\title{
THE ADDITIVE UNIT STRUCTURE OF COMPLEX BIQUADRATIC FIELDS
}

\author{
VOLKER ZIEGLER \\ Graz University of Technology, Austria
}

\begin{abstract}
We determine which rings of the form $\mathbb{Z}[\alpha]$ are generated by their units, where $\alpha$ is a root of the polynomial $X^{4}-B X^{2}+D$ such that $\alpha$ and all its conjugates are complex.
\end{abstract}

\section{INTRODUCTION}

Zelinsky [12] was the first who investigated the additive unit structure of rings, i.e. Zelinsky proved, if $V$ is a vector space over a division ring $D$, then every linear transformation can be written as the sum of two automorphisms unless $\operatorname{dim} V=1$ and $D$ is the field of two elements. Zelinsky's work gave rise to many investigations of rings that are generated by their units (see [10] for an overview). These investigations led Goldsmith, Pabst and Scott [5] to the following definition:

Definition 1.1. Let $R$ be a ring (with identity). An element $r$ is called $k$-good if $r=e_{1}+\cdots+e_{k}$, with $e_{1}, \ldots, e_{k} \in R^{*}$. If every element of $R$ is $k$-good we call also the ring $k$-good.

The unit sum number $u(R)$ is defined as $\min \{k: R$ is $k$-good $\}$. If the minimum does not exist but the units generate $R$ additively we set $u(R)=\omega$. If the units do not generate $R$ we set $u(R)=\infty$.

Although this topic has a history of more than 50 years, the interest in algebraic integers was marginal. In 1964 Jacobson [6] asked which quadratic fields have the property that every (algebraic) integer can be written as the

2000 Mathematics Subject Classification. 11R16, 11R27, 11A67.

Key words and phrases. Biquadratic fields, unit sum number.

The author gratefully acknowledges support from the Austrian Science Fund (FWF) under project Nr. P18079-N12. 
sum of distinct units. The problem was solved by Śliwa [8]. The cubic and quartic case was considered by Belcher [2,3]. Moreover, Belcher [2] characterized all quadratic fields whose rings of integers are generated by their units. About thirty years later Ashrafi and Vámos [1], Jarden and Narkiewicz [7] and Tichy and Ziegler [9] resumed this topic. In particular Ashrafi and Vámos showed that the ring of integers of quadratic fields, complex cubic fields and fields of the form $\mathbb{Q}\left(\zeta_{2^{n}}\right)$, with $\zeta_{2^{n}}$ is a $2^{n}$-th primitive root of unity, do not have finite unit sum number. This was generalized to all number fields by Jarden and Narkiewicz. Tichy and Ziegler [9] characterized all purely cubic number fields whose ring of integers are generated by their units. The case of purely complex quartic fields was solved by Filipin, Tichy and Ziegler [4]. Note that the case of quadratic fields has been rediscovered by Ashrafi and Vámos [1].

For the rest of the paper we assume $f(X)=X^{4}-B X^{2}+D$ is irreducible and all its roots are complex, i.e. $4 D>B^{2}$ or $-B, D>0$. Let $\alpha$ be one of the roots of $f(X)$. Then we investigate the additive unit structure of $\mathbb{Z}[\alpha]$. In particular we prove the following theorem.

THEOREM 1.2. $\mathbb{Z}[\alpha]$ is generated by its units if and only if $D= \pm 1$ or $B, D$ fulfill one of the cases listed in Table 1. In particular, if $\mathbb{Z}[\alpha]$ is generated by its units, then $D= \pm 1$ or $B^{2}-4 D=-4,-3$.

TABLE 1. List of unit bases for $\mathbb{Z}[\alpha]$, where $\rho=\frac{-1+\sqrt{-3}}{2}$ and $i=\sqrt{-1}$.

\begin{tabular}{|c|c||c|c|}
\hline$B$ & $D$ & $\epsilon$ & Basis \\
\hline \hline $2 n^{2}$ & $n^{4}+1$ & $n+\alpha$ & $\{1, i, \epsilon, \epsilon i\}$ \\
\hline$-2 n^{2}$ & $n^{4}+1$ & $n^{3}+\alpha+n \alpha^{2}$ & $\{1, i, \epsilon, \epsilon i\}$ \\
\hline $2 n^{2}+1$ & $n^{4}+n^{2}+1$ & $n+\alpha$ & $\{1, \rho, \epsilon, \epsilon \rho\}$ \\
\hline $2 n^{2}-1$ & $n^{4}-n^{2}+1$ & $n+\alpha$ & $\{1, \rho, \epsilon, \epsilon \rho\}$ \\
\hline$-6 n^{2}+1$ & $9 n^{4}-3 n^{2}+1$ & $\left(3 n^{3}-2 n\right)+2 n^{2} \alpha+n \alpha^{2}+\alpha^{3}$ & $\{1, \rho, \epsilon, \epsilon \rho\}$ \\
\hline$-6 n^{2}-1$ & $9 n^{4}+3 n^{2}+1$ & $\left(3 n^{3}+2 n\right)+2 n^{2} \alpha+n \alpha^{2}+\alpha^{3}$ & $\{1, \rho, \epsilon, \epsilon \rho\}$ \\
\hline-3 & 3 & $1+\alpha+\alpha^{3}$ & $\{1, \rho, \epsilon, \epsilon \rho\}$ \\
\hline
\end{tabular}

In Table 1 we denote by $n$ an arbitrary integer. The case $D= \pm 1$ can be excluded in our further investigations, since in this case $\alpha$ is a unit and $\mathbb{Z}[\alpha]$ is by trivial reasons generated by its units. The case $D=0$ can also be ignored, since otherwise $f(X)$ is reducible.

Before we start with the proof of the theorem, we have to determine the unit structure of the rings under consideration, in particular we have to determine, which roots of unity may appear (see section 2). The proof of Theorem 1.2 is divided into two cases. In the first case we assume that the 
"discriminant" $\Delta:=B^{2}-4 D$ is positive. In this case it turns out that the field $\mathbb{Q}(\alpha)$ is a CM-field, i.e. a totally complex field which is the quadratic extension of a totally real field. Since in CM-fields the unit structure is well known we will succeed in this case (see section 3 ). In section 4 we are concerned with the case of $\Delta<0$. In this case we will see that the Diophantine equation $X^{2}-\Delta Y^{2}= \pm 4$ is closely related to our problem and leads us to systems of equations, which are solved by using Groebner bases. In the last section we discuss some corollaries to Theorem 1.2.

In tables we assume that the signs must not be mixed, i.e. in one row we have to choose always the upper case sign or the lower case sign for all entries. In some tables, especially if we list units that generate rings of integers, mixed signs are allowed. Those tables are labeled with "mixed signs".

\section{RoOTS OF UNITY AND UNIT BASES}

In this section we determine in which cases roots of unity appear. By $\zeta_{n}$ we denote a primitive $n$-th root of unity. Before we start to investigate the unit structure we have to determine the Galois group of the polynomial $X^{4}-B X^{2}+D$.

LEMMA 2.1. The polynomial $X^{4}-B X^{2}+D$ is reducible if and only if $B^{2}-4 D$ is a square or $D=d^{2}$ and $B=b^{2}-2 d$ for some integers $b$ and $d$.

Assume $X^{4}-B X^{2}+D$ is irreducible. The Galois group $G$ corresponding to $X^{4}-B X^{2}+D$ is $\mathbb{Z}_{2} \times \mathbb{Z}_{2}$ if and only if $D$ is a square in $\mathbb{Z}, \mathbb{Z}_{4}$ if and only if $D=\frac{B^{2}}{4+k^{2}}$ with some $k \in \mathbb{Q}, D$ not a square in $\mathbb{Z}$ and $D_{4}$ otherwise. In the case $D$ not a square, $M=\mathbb{Q}\left(\sqrt{B^{2}-4 D}\right)$ is the unique quadratic subfield of $\mathbb{Q}(\alpha)$.

Proof. First, we prove the irreducibility statement. If $B^{2}-4 D$ is a square it is obvious that $X^{4}-B X^{2}+D$ is reducible. Therefore we assume $B^{2}-4 D$ is not a square. Assume $X^{4}-B X^{2}+D$ is reducible. Since $B^{2}-4 D$ is not a square no root of $X^{4}-B X^{2}+D$ is a rational. Therefore we have

$$
X^{4}-B X^{2}+D=\left(X^{2}+b_{1} X+d_{1}\right)\left(X^{2}+b_{2} X+d_{2}\right),
$$

with $b_{1}, b_{2}, d_{1}, d_{2} \in \mathbb{Z}$. By comparing coefficients we find $b_{1}=-b_{2}=b$. Note that $b \neq 0$ since otherwise $X^{2}-B X+D$ is reducible which yields $B^{2}-4 D$ is a square. Moreover, we find $B=b^{2}-d_{1}-d_{2}, 0=b_{2} d_{1}+b_{1} d_{2}$ and $D=d_{1} d_{2}$, i.e. $d_{1}=d_{2}=d, D=d^{2}$ and $B=b^{2}-2 d$.

Next, we compute the Galois group. We note that the only transitive subgroups of $S_{4}$ are $S_{4}, A_{4}, D_{4}, \mathbb{Z}_{4}$ and $\mathbb{Z}_{2} \times \mathbb{Z}_{2}$ up to conjugacy classes. Let $\alpha_{1}, \ldots, \alpha_{4}$ be the roots of $X^{4}-B X^{2}+D$ in a suitable order. Then we have

$$
\alpha_{1}+\alpha_{2}=\alpha_{3}+\alpha_{4}=0
$$

and $\alpha_{i}+\alpha_{j} \neq 0$ for $i<j$ and $(i, j) \neq(1,2),(3,4)$. Note that the cyclic permutation $(1,2,3)$ of the indices of $\alpha$ does not induce an automorphism. 
Therefore $G=\operatorname{Gal}(\mathbb{Q}(\alpha), \mathbb{Q})$ is not $S_{4}$ nor $A_{4}$. The only cyclic permutations of length 4 , which leave $(2.1)$ fixed, are $(1,3,2,4)$ and $(1,4,2,3)$. Moreover, in the case $\alpha_{1} \alpha_{3}=\sqrt{D} \in \mathbb{Z}$, the field $\mathbb{Q}(\alpha)$ is Galois and the Galois group cannot be cyclic, since the only admissible cyclic permutations yield automorphisms with $\sqrt{D} \mapsto-\sqrt{D}$. Note that the Galois group $\mathbb{Z}_{2} \times \mathbb{Z}_{2}$ is exactly the transitive group such that $(2.1)$ and $\alpha_{1} \alpha_{3}$ stays invariant. If $\sqrt{D} \in \mathbb{Q}(\alpha) \backslash \mathbb{Z}$ then $\mathbb{Q}(\alpha)$ is Galois and there exists an automorphism with $\sqrt{D} \mapsto-\sqrt{D}$, i.e. $G \neq \mathbb{Z}_{2} \times \mathbb{Z}_{2}$ and therefore $G=\mathbb{Z}_{4}$. In the case of $\sqrt{D} \notin \mathbb{Q}(\alpha)$ the field $\mathbb{Q}(\alpha)$ is not Galois and therefore has Galois group $D_{4}$.

If $D$ is not a square in $\mathbb{Z}$ then $\mathbb{Q}(\alpha)$ has Galois group $\mathbb{Z}_{4}$ or $D_{4}$. It is an immediate consequence of Galois theory that fields with such Galois groups have a unique quadratic subfield. On the other hand $\mathbb{Q}\left(\sqrt{B^{2}-4 D}\right)$ is a quadratic subfield of $\mathbb{Q}(\alpha)$, hence it is unique for $D \notin \mathbb{Z}^{2}$. Furthermore if $G=\mathbb{Z}_{4}$ we have $\mathbb{Q}(\sqrt{D})=\mathbb{Q}\left(\sqrt{B^{2}-4 D}\right)$ which yields $k^{2} D=B^{2}-4 D$ for some $k \in \mathbb{Q}$.

Let $\zeta_{n}$ be a primitive $n$-th root of unity. We prove:

Proposition 2.2. The field $\mathbb{Q}(\alpha)$ contains fourth and sixth roots of unity respectively in the following cases:

- If $B^{2}-4 D=-4 m^{2}$ with $m \in \mathbb{Z}$, then $\zeta_{4}=\frac{-B+2 \alpha^{2}}{2 m}$;

- If $B^{2}-4 D=-3 m^{2}$ with $m \in \mathbb{Z}$, then $\zeta_{6}=\frac{m-B+2 \alpha^{2}}{2 m}$;

- If $D=d^{2}$ and $B+2 d=-m^{2}$, with $d, m \in \mathbb{Z}$, then $\zeta_{4}=\frac{-(B+d) \alpha+\alpha^{3}}{B m}$ provided $B \neq 0$ and $\zeta_{4}=\frac{d \alpha-\alpha^{3}}{d m}$ otherwise;

- If $D=d^{2}$ and $B+2 d=-3 m^{2}$, with $d, m \in \mathbb{Z}$, then $\zeta_{6}=$ $\frac{B m-(B+d) \alpha+\alpha^{3}}{2 B m}$ provided $B \neq 0$ and $\zeta_{6}=\frac{d m+d \alpha-\alpha^{3}}{2 d m}$ otherwise.

Before we prove the proposition let us remark that the case $m=0$ is impossible, since otherwise we obtain in all cases $B^{2}-4 D=0$. But this implies $X^{4}-B X^{2}+D$ is reducible.

Proof. Let us assume $D$ is not a perfect square and $\zeta_{n} \in \mathbb{Q}(\alpha)$ with $n=4,6$. Then the only quadratic subfield of $\mathbb{Q}(\alpha)$ is $\mathbb{Q}\left(\sqrt{B^{2}-4 D}\right)=\mathbb{Q}\left(\zeta_{n}\right)$. We consider the case $n=4$ first. Therefore we conclude $B^{2}-4 D=-m^{\prime 2}$ or equivalently $B^{2}+m^{\prime 2}=4 D$. Assuming $m^{\prime}$ odd, we obtain $0 \equiv B^{2}+m^{\prime 2} \equiv 1,2$ $\bmod 4$ which is a contradiction. Therefore $m^{\prime}=2 m$ and we have $B^{2}-4 D=$ $-4 m^{2}$. In the case of $n=6$ we obtain $B^{2}-4 D=-3 m^{2}$ similarly.

Now let us assume $D=d^{2}$. Then there are 3 non-isomorphic quadratic subfields of $\mathbb{Q}(\alpha)$. These are beside $\mathbb{Q}\left(\sqrt{B^{2}-4 D}\right)$ the fields $\mathbb{Q}(\sqrt{B \pm 2 d})$. If $\zeta_{n} \notin \mathbb{Q}\left(\sqrt{B^{2}-4 D}\right)$, then $\zeta_{n} \in \mathbb{Q}(\sqrt{B+2 d})$. Indeed, if $\zeta_{n} \notin \mathbb{Q}(\sqrt{B+2 d})$ then make the change $d \rightarrow-d$. The case $\zeta_{n} \in \mathbb{Q}\left(\sqrt{B^{2}-4 D}\right)$ has been treated above. Therefore we conclude $B+2 d=-m^{2},-3 m^{2}$ depending on $n$. 
Now we have to compute the roots of unity. Note that $\alpha= \pm \sqrt{\frac{B \pm \sqrt{B^{2}-4 D}}{2}}$. Therefore we have $-B+2 \alpha^{2}= \pm \sqrt{B^{2}-4 D}$. If we substitute $B^{2}-4 D=$ $-4 m^{2},-3 m^{2}$ respectively we obtain the first two cases. Note that $\pm \sqrt{-1}$ and $(1 \pm \sqrt{-3}) / 2$ is for either sign a fourth and sixth root of unity respectively.

Now assume $D=d^{2}$ and $B+2 d=-m^{2},-3 m^{2}$. In this case we can write

$$
\alpha=\left(e_{1} \sqrt{B+2 d}+e_{2} \sqrt{B-2 d}\right) / 2,
$$

with $e_{1}, e_{2} \in\{ \pm 1\}$. By a simple computation we find

$$
\alpha^{3}=\left(e_{1}(B-d) \sqrt{B+2 d}+e_{2}(B+d) \sqrt{B-2 d}\right) / 2 .
$$

Therefore $\frac{-(B+d) \alpha+\alpha^{3}}{B}=e_{1} \sqrt{B+2 d}$ provided $B \neq 0$. Inserting $B+2 d=-m^{2}$ and $B+2 d=-3 m^{2}$ yields the third and fourth statement of the proposition respectively. The case $B=0$ can be proved similarly.

If $\zeta_{n} \in \mathbb{Q}(\alpha)$ we have $\phi(n) \mid 4$, i.e. $n=1,2,3,4,5,6,8,10,12$. Since for $n$ odd $\zeta_{2 n}=-\zeta_{n}$ it suffices to consider the case $n$ is even.

Proposition 2.3. We have the following relations:

$$
\begin{aligned}
\zeta_{4} & \in \mathbb{Z}[\alpha] \Longleftrightarrow B^{2}-4 D=-4 \text { or } B=D=1 ; \\
\zeta_{6} & \in \mathbb{Z}[\alpha] \Longleftrightarrow B^{2}-4 D=-3 ; \\
\zeta_{8} & \in \mathbb{Z}[\alpha] \Longleftrightarrow B=0, D=1 ; \\
\zeta_{12} & \in \mathbb{Z}[\alpha] \Longleftrightarrow B=D=1 .
\end{aligned}
$$

Moreover, $\zeta_{10} \notin \mathbb{Z}[\alpha]$ for any $\alpha$.

Proof. Assume $\zeta_{4} \in \mathbb{Z}[\alpha]$. Because of Proposition 2.2 either $B^{2}-4 D=$ $-4 m^{2}$, with $m= \pm 1$ or $B+2 d=-m^{2}$, where $B m= \pm 1$. In the first case we obtain $B^{2}-4 D=-4$ and in the second case we get $\pm 1+2 d=-1$, i.e $B=-1$ and $d=0$ or $B=1$ and $d=1$. This yields the first statement. Note that $d=0$ yields $D=0$ which has been excluded.

If $\zeta_{6} \in \mathbb{Z}[\alpha]$, then we have $B^{2}-4 D=-3 m^{2}$ with $m= \pm 1$ or $B+2 d=$ $-3 m^{2}$ with $2 B m= \pm 1$. The first case yields $B^{2}-4 D=-3$ and the second case is a contradiction.

In the case of $n=8,10,12$ we have $\mathbb{Q}\left(\zeta_{n}\right)=\mathbb{Q}(\alpha)$ and $\zeta_{n} \in \mathbb{Z}[\alpha]$ implies that $\mathbb{Z}[\alpha]$ is the maximal order. Computing discriminants we see that $\delta_{\mathbb{Z}[\alpha]}=$ $16\left(B^{2}-4 D\right)^{2} D$ and $\delta_{n}=2^{8}, 5^{3}, 2^{4} 3^{2}$ for $n=8,10,12$ where $\delta_{n}$ denotes the discriminant of $\mathbb{Q}\left(\zeta_{n}\right)$. Note if $\mathbb{Z}[\alpha]$ is the maximal order of $\mathbb{Q}(\alpha)=\mathbb{Q}\left(\zeta_{n}\right)$ we have $\delta_{\mathbb{Z}[\alpha]}=\delta_{n}$. For $n=8$ we deduce $\left(B^{2}-4 D\right)^{2} D=16$ which yields $B=0$ and $D=1$, for $n=10$ we have $16 \mid 5^{3}$, a contradiction, and for $n=12$ we obtain $\left(B^{2}-4 D\right)^{2} D=9$ which yields $B=D=1$.

Next we investigate possible integral bases for $\mathbb{Z}[\alpha]$ which consist of units. We prove the following version of [9, Lemma 1]: 
Lemma 2.4. Let $\alpha$ be an algebraic integer of degree 4, totally complex, i.e all its conjugates are complex, and let $\zeta \in \mathbb{Z}[\alpha]$ be an $n$-th root of unity, with $n$ maximal. Then the ring $\mathbb{Z}[\alpha]$ is generated by its units if and only if it is generated by $\left\{1, \zeta, \zeta^{2}, \ldots, \zeta^{n-1}, \epsilon, \zeta \epsilon, \ldots, \zeta^{n-1} \epsilon^{3}\right\}$, where $\epsilon$ is the fundamental unit of $\mathbb{Z}[\alpha]$.

Proof. By Dirichlet's unit theorem we may assume $\mathbb{Z}[\alpha]$ is generated by

$$
\left\{\zeta^{k_{1}} \epsilon^{l_{1}}, \zeta^{k_{2}} \epsilon^{l_{2}}, \zeta^{k_{3}} \epsilon^{l_{3}}, \zeta^{k_{4}} \epsilon^{l_{4}}\right\} .
$$

Since $\epsilon$ is an algebraic integer of degree four, $\zeta^{k} \epsilon^{l}$ can be written as a linear combination of $\zeta^{k}, \zeta^{k} \epsilon, \zeta^{k} \epsilon^{2}, \zeta^{k} \epsilon^{3}$, which already proves the lemma.

Proposition 2.5. Let $|D|>1$, and assume $\mathbb{Z}[\alpha]$ is generated by its units. Then there exists a unit $\epsilon \in \mathbb{Z}[\alpha]^{*}$ such that:

- The basis $\left\{1, \epsilon, \epsilon^{2}, \epsilon^{3}\right\}$ generates $\mathbb{Z}[\alpha]$ if $B^{2}-4 D \neq-3,-4$;

- The basis $\left\{1, \zeta_{6}, \epsilon, \epsilon \zeta_{6}\right\}$ generates $\mathbb{Z}[\alpha]$ if $B^{2}-4 D=-3$;

- The basis $\left\{1, \zeta_{4}, \epsilon, \epsilon \zeta_{4}\right\}$ generates $\mathbb{Z}[\alpha]$ if $B^{2}-4 D=-4$.

Moreover, in each case the basis, where $\epsilon$ is replaced by $\epsilon^{-1}$, generates also $\mathbb{Z}[\alpha]$.

Proof. By Proposition 2.3 we know, if $|D|>1$ roots of unity other than \pm 1 occur in $\mathbb{Z}[\alpha]$ if and only if $B^{2}-4 D=-3,-4$. Let us assume $B^{2}-4 D \neq-3,-4$. Since $\mathbb{Q}(\alpha)$ is totally complex and of degree four we have by Dedekind's unit theorem, that all units are of the form $\pm \epsilon^{k}$, where $\epsilon$ is a fundamental unit. Therefore the first case is proved.

Now assume $B^{2}-4 D=-4,-3$. By Lemma 2.4 we know if $\mathbb{Z}[\alpha]$ is generated by its units then there exists a subset of $E=\left\{1, \zeta, \epsilon, \epsilon \zeta, \epsilon^{2}, \epsilon^{2} \zeta, \epsilon^{3}, \epsilon^{3} \zeta\right\}$ with four elements which is a basis for $\mathbb{Z}[\alpha]$. By $\zeta$ we denote $\zeta_{4}, \zeta_{6}$ depending on $B^{2}-4 D=-4,-3$. Indeed, since $\zeta_{6}^{3}=\zeta_{4}^{2}=-1$ and $\zeta_{6}^{2}=\zeta_{6}-1$ we only have to consider subsets of $E$. A subset $\mathcal{B}=\left\{\epsilon_{1}, \epsilon_{2}, \epsilon_{3}, \epsilon_{4}\right\}$ is a basis of $\mathbb{Z}[\alpha]$ if and only if the Matrix $M$ corresponding to the base change from $\mathcal{B}$ to $\left\{1, \alpha, \alpha^{2}, \alpha^{3}\right\}$ has determinant \pm 1 . Let us write $\epsilon=x+y \alpha+z \alpha^{2}+w \alpha^{3}$. Moreover let $\epsilon_{i}=x_{i}+y_{i} \alpha+z_{i} \alpha^{2}+w_{i} \alpha^{3} \in E$, then $\mathbb{Z}[\alpha]$ has basis $\left\{\epsilon_{1}, \epsilon_{2}, \epsilon_{3}, \epsilon_{4}\right\}$ if and only if

$$
\operatorname{det} M=\operatorname{det}\left(\begin{array}{cccc}
x_{1} & y_{1} & z_{1} & w_{1} \\
x_{2} & y_{2} & z_{2} & w_{2} \\
x_{3} & y_{3} & z_{3} & w_{3} \\
x_{4} & y_{4} & z_{4} & w_{4}
\end{array}\right)= \pm 1 .
$$

We compute the determinant for all 70 possible cases and recognize that each determinant has the factor $D w^{2}+B w y+y^{2}$. This yields in any case $D w^{2}+B w y+y^{2}= \pm 1$. But the determinant corresponding to the basis $\{1, \zeta, \epsilon, \epsilon \zeta\}$ is exactly $D w^{2}+B w y+y^{2}$. Therefore the cases $B^{2}-4 D=3,4$ are established. 
Now we prove the last statement of the proposition. Obviously a basis of $\mathbb{Z}[\alpha]$ remains a basis if each of its elements is multiplied by the same unit $\epsilon$. Therefore we conclude, if $\left\{1, \epsilon, \epsilon^{2}, \epsilon^{3}\right\}$ is a basis then $\left\{\epsilon^{-3}, \epsilon^{-2}, \epsilon^{-1}, 1\right\}$ (multiplication by $\epsilon^{-3}$ ) is also a basis. Similar, if $\{1, \zeta, \epsilon, \epsilon \zeta\}$ is a basis, then $\left\{\epsilon^{-1}, \epsilon^{-1} \zeta, 1, \zeta\right\}$ (multiplication by $\epsilon^{-1}$ ) is also a basis.

\section{THE CASE $B^{2}-4 D>0$}

This section is devoted to the case $B^{2}-4 D>0$. Therefore $\mathbb{Q}(\alpha)$ is a CMfield, i.e. a field that is a totally complex extension of a totally real number field. The following theorem yields a tool to prove Theorem 1.2 in this case (see [11, Theorem 4.12]).

Theorem 3.1. Let $K$ be a $C M$-field and let $R$ be an order of $K, E$ the unit group of $R, K^{+}$the maximal real subfield of $K, E^{+}$the unit group of $R \cap K^{+}$and $W$ the roots of unity lying in $R$. Then

$$
\left[E: W E^{+}\right]=1,2 \text {. }
$$

Proof. The proof of this theorem can be taken word by word from [11, Theorem 4.12]. Note that Washington proved this theorem in the case of $R$ is the maximal order but this property is not needed.

Because of Proposition 2.3 we know that $\mathbb{Z}[\alpha]$ contains no roots of unity other than \pm 1 . Let $\epsilon \in \mathbb{Z}[\alpha]^{*}$, then we conclude by Theorem 3.1 that either $\epsilon$ or $\epsilon^{2}$ is real. In the case $\epsilon$ is real obviously $\mathbb{Z}[\alpha]$ is not generated by $1, \epsilon, \epsilon^{2}$ and $\epsilon^{3}$. Therefore we assume that $\epsilon$ is not real but $\epsilon^{2}$ is real. Let us write

$$
\epsilon=x+y \alpha+z \alpha^{2}+w \alpha^{3} .
$$

We compute

$$
\begin{aligned}
\epsilon^{2}=\left(x^{2}-\right. & \left.2 D w y-D z^{2}-B D w^{2}\right)+2(x y-D w z) \alpha \\
& +\left((B w+y)^{2}+B z^{2}+2 x z-D w^{2}\right) \alpha^{2}+2(w x+B w z+y z) \alpha^{3} .
\end{aligned}
$$

Since $\alpha$ and $\alpha^{3}$ are purely imaginary and linear independent we conclude

$$
x y=D w z, \quad w x+B w z+y z=0 .
$$

Multiplying the second equation by y yields $z\left(D w^{2}+B w y+y^{2}\right)=0$. Since $D w^{2}+B w y+y^{2}=0$ implies $\mathbb{Q}(\alpha)$ is not quartic, we deduce $z=0$, hence $w x=x y=0$. Therefore either $x=0$ or $w=y=0$. In the case of $x=0$, we deduce that $\alpha$ is a unit, hence $D= \pm 1$. In the case of $w=y=0$ we get $\epsilon=x= \pm 1$. But obviously \pm 1 does not generate $\mathbb{Z}[\alpha]$. 


\section{The CASE of $B^{2}-4 D<-4$}

Because of Proposition 2.5 (see also [9, Lemma 1]) we may assume $\left\{1, \epsilon, \epsilon^{2}, \epsilon^{3}\right\}$ generates $\mathbb{Z}[\alpha]$. Let the notations be as above and write

$$
\begin{aligned}
& \epsilon^{2}=x_{2}+y_{2} \alpha+z_{2} \alpha^{2}+w_{2} \alpha^{3}, \\
& \epsilon^{3}=x_{3}+y_{3} \alpha+z_{3} \alpha^{2}+w_{3} \alpha^{3},
\end{aligned}
$$

with

$$
\begin{gathered}
x_{2}=-B D w^{2}+x^{2}-2 D w y-D z^{2}, \quad y_{2}=2 x y-2 D w z \\
z_{2}=B^{2} w^{2}-D w^{2}+2 B w y+y^{2}+2 x z+B z^{2}, \quad w_{2}=2 w x+2 B w z+2 y z
\end{gathered}
$$

and

$$
\begin{aligned}
x_{3}= & x\left(x^{2}-3 D w(B w+2 y)\right)-3 D z\left(w^{2}\left(B^{2}-D\right)+2 B w y+y^{2}\right) \\
& -3 D x z^{2}-B D z^{3} \\
y_{3}= & D^{2} w^{3}-B^{2} D w^{3}+3 x^{2} y-3 B D w\left(w y+z^{2}\right) \\
& -3 D\left(w y^{2}+2 w x z+y z^{2}\right), \\
z_{3}= & 3 x\left(w^{2}\left(B^{2}-D\right)+2 B w y+y^{2}\right)+3 z\left(x^{2}+(B w+y)\left(B^{2} w-2 D w+B y\right)\right) \\
& +3 B x z^{2}+z^{3}\left(B^{2}-D\right), \\
w_{3}= & B^{3} w^{3}-2 B D w^{3}+3 w x^{2}+3 B^{2} w^{2} y-3 D w^{2} y+3 B w y^{2}+y^{3} \\
& +6 x z(B w+y)+3 z^{2}\left(B^{2} w-D w+B y\right) z .
\end{aligned}
$$

Then $\left\{1, \epsilon, \epsilon^{2}, \epsilon^{3}\right\}$ generates $\mathbb{Z}[\alpha]$ if and only if

$$
\operatorname{det} M=\operatorname{det}\left(\begin{array}{cccc}
1 & 0 & 0 & 0 \\
x & y & z & w \\
x_{2} & y_{2} & z_{2} & w_{2} \\
x_{3} & y_{3} & z_{3} & w_{3}
\end{array}\right)= \pm 1
$$

(see [9] or proof of Proposition 2.5). A computation shows

$$
\begin{aligned}
\operatorname{det} M= & \left(\left(y+B \frac{w}{2}\right)^{2}-\frac{\Delta}{4} w^{2}\right) \times \\
& \left(\left(\left(B^{2}-D\right) w^{2}+2 B w y+y^{2}\right)^{2}+\left(B^{2}-4 D\right) z^{4}\right. \\
& \left.-2\left(B\left(B^{2}-3 D\right) w^{2}+2\left(B^{2}-2 D\right) w y+B y^{2}\right) z^{2}\right),
\end{aligned}
$$

i.e. $(2 y+B w)^{2}-\Delta w^{2}= \pm 4$. Since $\Delta=B^{2}-4 D<-4$, we conclude $w=0$ and $y= \pm 1$. But $\left\{1, \epsilon, \epsilon^{2}, \epsilon^{3}\right\}$ is a basis and therefore $\left\{1, \epsilon^{-1}, \epsilon^{-2}, \epsilon^{-3}\right\}$ generates also $\mathbb{Z}[\alpha]$. Because of $w=0, y= \pm 1$ and $\epsilon$ a unit we obtain

$$
\epsilon^{-1}=x_{-1}+y_{-1} \alpha+z_{-1} \alpha^{2}+w_{-1} \alpha^{3},
$$


with $w_{-1}=0$ and $y_{-1}= \pm 1$. On the other hand we have

$$
\begin{gathered}
x_{-1}=-B x+x^{3}-D z+2 B x^{2} z+\left(B^{2}+D\right) x z^{2}+B D z^{3}, \\
y_{-1}= \pm\left(B-x^{2}-2 B x z-B^{2} z^{2}+D z^{2}\right), \quad z_{-1}=x-x^{2} z-B x z^{2}-D z^{3} \\
w_{-1}= \pm\left(B z^{2}+2 x z-1\right) .
\end{gathered}
$$

Since we have $w_{-1}=0$, we find $1=z(B z+2 x)$, which yields $z=e= \pm 1$ and $x=e(1-B) / 2$. Furthermore, we obtain $y_{-1}=\frac{(B-1)^{2}-4 D}{4}= \pm 1$. Therefore $B=1 \pm 2 \sqrt{ \pm 1+D}$ and we have $B=1+e_{1} 2 n$ and $D=n^{2}+e_{2}$ with $n \in \mathbb{Z}$ and $e_{1}, e_{2} \in\{ \pm 1\}$. Therefore we conclude $\epsilon= \pm\left(n+\alpha^{2}\right) \pm \alpha$ with mixed signs. But a short computation shows that in this case we have $|\operatorname{det} M|=4 \neq 1$, a contradiction.

\section{THE CASES $B^{2}-4 D=-3,-4$}

We start with the case $B^{2}-4 D=-4$. Obviously $B$ has to be even and we write $B=2 b$ and $D=b^{2}+1$. By the proof of Proposition 2.5 we know that we have to investigate the equation $D w^{2}+B w y+y^{2}= \pm 1$, i.e. we have

$$
(y+b w)^{2}+w^{2}=1 .
$$

Therefore we conclude $w=0, y= \pm 1$ or $w=1, y=-b$ or $w=-1, y=b$. By Proposition 2.5 the coefficients of $\epsilon^{-1}$ have also to satisfy an analogous relation. Let us write $\epsilon^{-1}=x_{-1}+y_{-1} \alpha+z_{-1} \alpha^{2}+w_{-1} \alpha^{3}$. The formulas for $y_{-1}$ and $w_{-1}$ are computed in Table 2 .

Hence we obtain 16 systems of equations. For each system we compute a Groebner basis (see Table 3) with respect to the lexicographic term order induced by $x \succ z \succ b$.

TABLE 2. The values of $y_{-1}$ and $w_{-1}$ in the case of $B^{2}-4 D=-4$.

\begin{tabular}{|c|c||c|}
\hline$y$ & $w$ & $y_{-1}$ and $w_{-1}$ \\
\hline \hline 1 & 0 & $\begin{array}{l}y_{-1}=-x^{2}+z^{2}-3 b^{2} z^{2}+b(2-4 x z) \\
w_{-1}=-1+2 x z+2 b z^{2}\end{array}$ \\
\hline-1 & 0 & $\begin{array}{l}y_{-1}=x^{2}-z^{2}+3 b^{2} z^{2}-b(2-4 x z) \\
w_{-1}=1-2 x z-2 b z^{2}\end{array}$ \\
\hline$-b$ & 1 & $\begin{array}{l}y_{-1}=-1-2 x z+b^{3} z^{2}+b^{2}(1+2 x z)+b\left(x^{2}-3 z^{2}\right) \\
w_{-1}=-b-x^{2}-2 b x z+z^{2}-b^{2} z^{2}\end{array}$ \\
\hline$b$ & -1 & $\begin{array}{l}y_{-1}=1+2 x z-b^{3} z^{2}-b^{2}(1+2 x z)-b\left(x^{2}-3 z^{2}\right) \\
w_{-1}=b+x^{2}+2 b x z-z^{2}+b^{2} z^{2}\end{array}$ \\
\hline
\end{tabular}

The first four Groebner bases do not yield solutions since otherwise we would obtain $4 \mid 1$. The last two Groebner bases yield $b= \pm\left(z^{2}-1 / z^{2}\right)$, hence $z^{2}=1$ and therefore $b=0$ and $D=-1$ which yields a trivial case. The fifth (sixth) Groebner basis yields either $z=0$ and $b=x^{2}\left(z=0\right.$ and $\left.b=-x^{2}\right)$ or 
TABLE 3. The Groebner bases.

\begin{tabular}{|c|c|c|c||c|}
\hline$y$ & $w$ & $y_{-1}$ & $w_{-1}$ & Groebner basis \\
\hline \hline \pm 1 & 0 & \pm 1 & 0 & $1+4 z^{2}-4 b z^{2}-4 z^{4}, x+2 z-b z-2 z^{3}$ \\
\pm 1 & 0 & $\mp 1$ & 0 & $1-4 z^{2}-4 b z^{2}-4 z^{4}, x-2 z-b z-2 z^{3}$ \\
$\pm b$ & $\mp 1$ & $\pm b$ & $\mp 1$ & $1+4 z^{2}+4 b z^{2}-4 z^{4}, x+2 z+b z-2 z^{3}$ \\
$\pm b$ & $\mp 1$ & $\mp b$ & \pm 1 & $1+4 z^{2}-4 b z^{2}+4 z^{4}, x+2 z-b z+2 z^{3}$ \\
\pm 1 & 0 & $\pm b$ & $\mp 1$ & $b z+z^{3}, x z+b z^{2}, b-x^{2}+z^{2}+b^{2} z^{2}$ \\
$\pm b$ & $\mp 1$ & \pm 1 & 0 & $b z-z^{3}, x z+b z^{2}, b+x^{2}-z^{2}-b^{2} z^{2}$ \\
\pm 1 & 0 & $\mp b$ & \pm 1 & $1-b z^{2}-z^{4}, x-z^{3}$ \\
$\pm b$ & $\mp 1$ & $\mp 1$ & 0 & $1+b z^{2}-z^{4}, x+z^{3}$ \\
\hline
\end{tabular}

$z \neq 0$ and $b=-z^{2}, x=z^{3}\left(z \neq 0\right.$ and $\left.b=z^{2}, x=-z^{3}\right)$. Let us put $x=n$ in the case $z=0$ and $z=n$ in the other case, where $n \in \mathbb{Z}$ is arbitrary. These two cases yield all possible $\epsilon$ for which $\mathbb{Z}[\alpha]$ is generated by $\left\{1, \zeta_{4}, \epsilon, \epsilon \zeta_{4}\right\}$. These $\epsilon$ are listed in Table 4. It is easy to check that in each case $\epsilon$ is a unit (one has to verify $\mathrm{N}_{K / \mathbb{Q}}(\epsilon)= \pm 1$ for each possible $\epsilon$ ).

TABLE 4. Units $\epsilon$ such that $\left\{1, \zeta_{4}, \epsilon, \epsilon \zeta_{4}\right\}$ generates $\mathbb{Z}[\alpha]$. (mixed signs)

\begin{tabular}{|c|c|c|}
\hline$B$ & $D$ & $\epsilon$ \\
\hline \hline $2 n^{2}$ & $n^{4}+1$ & $\pm\left(n^{3}+n \alpha^{2}\right) \pm\left(n^{2} \alpha-\alpha^{3}\right)$ \\
\hline$-2 n^{2}$ & $n^{4}+1$ & $\begin{array}{l} \pm n \pm\left(n^{2} \alpha+\alpha^{3}\right) \\
\pm\left(n^{3}+n \alpha^{2}\right) \pm \alpha\end{array}$ \\
\hline
\end{tabular}

Now we consider the case $B^{2}-4 D=-3$. In this case $B$ has to be odd and therefore we write $B=2 b-1$ and $D=b^{2}-b+1$. By Proposition 2.5 we have to investigate the equation $\operatorname{det} M=(y+w B / 2)^{2}+3 w^{2} / 4= \pm 1$ or equivalently

$$
(2 y+2 w b-w)^{2}+3 w^{2}= \pm 4 .
$$

Equation (5.2) has exactly 6 solutions namely

$$
(y, w)=( \pm 1,0),( \pm b, \mp 1),( \pm(1-b), \pm 1) .
$$

As in the case of $B^{2}-4 D=-4$ we know that the coefficients of $\epsilon^{-1}$ satisfy also relation $(5.2)$, i.e. $\left(2 y_{-1}+2 w_{-1} b-w_{-1}\right)^{2}+3 w_{-1}^{2}= \pm 4$. In order to find more relations we compute $y_{-1}$ and $w_{-1}$ (see Table 5 ).

Similar as in the case of $B^{2}-4 D=-4$ we find 36 systems of equations. We compute for each system a Groebner basis with respect to the lexicographic term order $x \succ z \succ b$. The first element of each Groebner basis is given in Table 6 . 
TABLE 5. The values of $y_{-1}$ and $w_{-1}$ in the case of $B^{2}-4 D=-3$.

\begin{tabular}{|c|c||c|}
\hline$y$ & $w$ & $y_{-1}$ and $w_{-1}$ \\
\hline \hline 1 & 0 & $\begin{array}{l}y_{-1}=-1+2 b-x^{2}+2 x z-4 b x z+3 b z^{2}-3 b^{2} z^{2} \\
w_{-1}=-1+2 x z+(2 b-1) z^{2}\end{array}$ \\
\hline-1 & 0 & $\begin{array}{l}y_{-1}=1-2 b+x^{2}-2 x z+4 b x z-3 b z^{2}+3 b^{2} z^{2} \\
w_{-1}=1-2 x z-(2 b-1) z^{2}\end{array}$ \\
\hline$-b$ & 1 & $\begin{array}{l}y_{-1}=b\left(b-2+x^{2}\right)+2\left(b^{2}-1\right) x z+\left(1-3 b+b^{3}\right) z^{2} \\
w_{-1}=1-b-x^{2}-2 b x z+z^{2}-b^{2} z^{2}\end{array}$ \\
\hline $1-b$ & 1 & $\begin{array}{l}y_{-1}=(b-1)\left(1+b+x^{2}\right)+2(b-2) b x z+\left(b^{3}-3 b^{2}+1\right) z^{2} \\
w_{-1}=x(2 z-x)-b\left(1+2 x z+(b-2) z^{2}\right)\end{array}$ \\
\hline$b$ & -1 & $\begin{array}{l}y_{-1}=b\left(2-b-x^{2}\right)-2\left(b^{2}-1\right) x z-\left(1-3 b+b^{3}\right) z^{2} \\
w_{-1}=x^{2}+b-1+2 b x z-z^{2}+b^{2} z^{2}\end{array}$ \\
\hline$b-1$ & -1 & $\begin{array}{l}y_{-1}=(1-b)\left(1+b+x^{2}\right)-2(b-2) b x z-\left(b^{3}-3 b^{2}+1\right) z^{2} \\
x(x-2 z)+b\left(1+2 x z+(b-2) z^{2}\right)\end{array}$ \\
\hline
\end{tabular}

TABLE 6. The first element of the Groebner bases.

\begin{tabular}{|c|c|c|c||c|}
\hline$y$ & $w$ & $y_{-1}$ & $w_{-1}$ & first element of the Groebner basis \\
\hline \hline \pm 1 & 0 & \pm 1 & 0 & $1+6 z^{2}-4 b z^{2}-3 z^{4}$ \\
\pm 1 & 0 & $\mp 1$ & 0 & $1-2 z^{2}-4 b z^{2}-3 z^{4}$ \\
\pm 1 & 0 & $\pm(1-b)$ & \pm 1 & $4+4 z^{2}-4 b z^{2}-3 z^{4}$ \\
\pm 1 & 0 & $\mp b$ & \pm 1 & $4-4 b z^{2}-3 z^{4}$ \\
\pm 1 & 0 & $\pm b$ & $\mp 1$ & $4 z-4 b z-3 z^{3}$ \\
\pm 1 & 0 & $\pm(b-1)$ & $\mp 1$ & $4 b z+3 z^{3}$ \\
$\pm b$ & $\mp 1$ & \pm 1 & 0 & $1-2 b+b^{2}-2 z^{2}+2 b z^{2}-3 z^{4}$ \\
$\pm b$ & $\mp 1$ & $\mp 1$ & 0 & $1+2 b+b^{2}-6 z^{2}+2 b z^{2}-3 z^{4}$ \\
$\pm b$ & $\mp 1$ & $\pm(1-b)$ & \pm 1 & $1-2 b+b^{2}-6 z^{2}+2 b z^{2}-3 z^{4}$ \\
$\pm b$ & $\mp 1$ & $\mp b$ & \pm 1 & $b^{2}-8 z^{2}+2 b z^{2}-3 z^{4}$ \\
$\pm b$ & $\mp 1$ & $\pm b$ & $\mp 1$ & $b^{2}+2 b z^{2}-3 z^{4}$ \\
$\pm b$ & $\mp 1$ & $\pm(b-1)$ & $\mp 1$ & $1+2 b+b^{2}-2 z^{2}+2 b z^{2}-3 z^{4}$ \\
$\pm(b-1)$ & $\mp 1$ & \pm 1 & 0 & $b^{2}+2 b z^{2}-3 z^{4}$ \\
$\pm(b-1)$ & $\mp 1$ & $\mp 1$ & 0 & $4-4 b+b^{2}+4 z^{2}+2 b z^{2}-3 z^{4}$ \\
$\pm(b-1)$ & $\mp 1$ & $\pm(1-b)$ & \pm 1 & $1-2 b+b^{2}-2 z^{2}+2 b z^{2}-3 z^{4}$ \\
$\pm(b-1)$ & $\mp 1$ & $\mp b$ & \pm 1 & $4-4 b+b^{2}+2 b z^{2}-3 z^{4}$ \\
$\pm(b-1)$ & $\mp 1$ & $\pm b$ & $\mp 1$ & $b^{2}+4 z^{2}+2 b z^{2}-3 z^{4}$ \\
$\pm(b-1)$ & $\mp 1$ & $\pm(b-1)$ & $\mp 1$ & $1-2 b+b^{2}+6 z^{2}+2 b z^{2}-3 z^{4}$ \\
\hline
\end{tabular}

Let us investigate the case $z=0$. In this case the first elements of the Groebner bases, get polynomials in $b$. Only in the cases $y= \pm 1, w=0$, $y_{-1}= \pm b, w_{-1}=\mp 1$ and $y= \pm 1, w=0, y_{-1}= \pm(b-1), w_{-1}=\mp 1$ the considered element vanishes unconditionally. In these cases the corresponding 
systems turn into

$$
\begin{array}{ll} 
\pm\left(-1+2 b-x^{2}\right)= \pm(b-1), & \mp 1=\mp 1, \\
\pm\left(-1+2 b-x^{2}\right)= \pm b, & \mp 1=\mp 1 .
\end{array}
$$

System (5.3) yields $b=x^{2}$ and (5.4) yields $b=x^{2}+1$. Let $x=n$, with $n \in \mathbb{Z}$ arbitrary, then we obtain $B=2 n^{2}-1, D=n^{4}+n^{2}+1$ and $\epsilon= \pm n \pm \alpha$ and $B=2 n^{2}+1, D=n^{4}-n^{2}+1$ and $\epsilon= \pm n \pm \alpha$ with mixed signs respectively. By computing norms we see that these $\epsilon$ 's are indeed units. See also Table 1.

In all other cases we deduce $b=-1,0,1$ or 2 . The case $b=0,1$ implies $D=-1$. Inserting $z=0, b=1$ and $z=0, b=2$ into Table 5 respectively yields the equations $1=0, x^{2} \pm 1=0$ and $x^{2} \pm 2=0$. Note that the equation $x^{2}-1=0$ appears only for $b=-1, y= \pm b, w=\mp 1, y_{-1}= \pm(b-1), w_{-1}=\mp 1$. Therefore we have $\epsilon= \pm 1 \pm\left(\alpha+\alpha^{3}\right)$ with mixed signs, if $-B=D=3$.

Now we may assume $z \neq 0$. In each case we compute the zeros of the first element of the Groebner bases (see Table 6) considered as polynomials in $b$. The solutions can be found in table 7 .

TABLE 7. Expressions for $b$.

\begin{tabular}{|c|c|c|c||c|}
\hline$y$ & $w$ & $y_{-1}$ & $w_{-1}$ & $b$ \\
\pm 1 & 0 & \pm 1 & 0 & $\frac{1}{4}\left(6+\frac{1}{\left.z^{2}-3 z^{2}\right)}\right.$ \\
\pm 1 & 0 & $\mp 1$ & 0 & $\frac{1}{4}\left(-2+\frac{1}{z^{2}}-3 z^{2}\right)$ \\
\pm 1 & 0 & $\mp b$ & \pm 1 & $1+\frac{1}{z^{2}}-\frac{3 z^{2}}{4}$ \\
\pm 1 & 0 & $\pm b$ & $\mp 1$ & $1-\frac{3 z^{2}}{4}$ \\
\pm 1 & 0 & $\pm(b-1)$ & $\mp 1$ & $-\frac{3 z^{2}}{4}$ \\
$\pm b$ & $\mp 1$ & \pm 1 & 0 & $1-3 z^{2}, 1+z^{2}$ \\
$\pm b$ & $\mp 1$ & $\mp 1$ & 0 & $-1-z^{2} \pm 2 z \sqrt{z^{2}+2}$ \\
$\pm b$ & $\mp 1$ & $\pm(1-b)$ & \pm 1 & $1-z^{2} \pm 2 z \sqrt{z^{2}+1}$ \\
$\pm b$ & $\mp 1$ & $\mp b$ & \pm 1 & $-z^{2} \pm 2 z \sqrt{z^{2}+2}$ \\
$\pm b$ & $\mp 1$ & $\pm b$ & $\mp 1$ & $-3 z^{2}, z^{2}$ \\
$\pm b$ & $\mp 1$ & $\pm(b-1)$ & $\mp 1$ & $-1-z^{2} \pm 2 z \sqrt{z^{2}+1}$ \\
$\pm(b-1)$ & $\mp 1$ & \pm 1 & 0 & $-3 z^{2}, z^{2}$ \\
$\pm(b-1)$ & $\mp 1$ & $\mp 1$ & 0 & $2-z^{2} \pm 2 z \sqrt{z^{2}-2}$ \\
$\pm(b-1)$ & $\mp 1$ & $\pm(1-b)$ & \pm 1 & $1-3 z^{2}, 1+z^{2}$ \\
$\pm(b-1)$ & $\mp 1$ & $\mp b$ & \pm 1 & $2-z^{2} \pm 2 z \sqrt{z^{2}-1}$ \\
$\pm(b-1)$ & $\mp 1$ & $\pm b$ & $\mp 1$ & $-z^{2} \pm 2 z \sqrt{z^{2}-1}$ \\
$\pm(b-1)$ & $\mp 1$ & $\pm(b-1)$ & $\mp 1$ & $1-z^{2} \pm 2 z \sqrt{z^{2}-2}$ \\
\hline
\end{tabular}

Table 7 shows that we have 18 different subcases. The first two cases yield $z^{2}=1$ since otherwise $b$ would not be an integer. Therefore we obtain $b=1$ and $b=-1$. But $b=1$ yields $D=1$ and we ignore this case. In the case 
of $b=-1$ the corresponding system turns into $\left(x-3 e_{1}\right)^{2}=1$ and $2 x e_{1}=4$, where $z=e_{1}$ with $e_{1} \in\{ \pm 1\}$. Therefore we find $x=2 e_{1}$. Indeed this solution yields a unit. Note that $b=-1$ implies $B=-3$ and $D=3$.

The cases 3 and 4 yield for integral $z$ no integral $b$, hence we have no solution.

Now let us consider the cases $5,6,7,11,13$ and 15 . In the cases $7,11,13$ and 15 we see that for any integral $z$ we obtain an integral $b$. Therefore we let $z=n$ where $n \in \mathbb{Z}$ is arbitrary. In the cases 5 and 6 we find that for every even $z$ we have an integral $b$. Therefore we put $z=2 n$ with $n \in \mathbb{Z}$. Inserting these results into the equations given by Table 5 we obtain systems of equations in $x$ and $n$. Solving for $x$ we find in each case an integral solution for $x$. These solutions yield indeed units. We list these solutions in Table 8 .

Now we consider the remaining cases. In each expression for $b$ we find the term $2 z \sqrt{z^{2}-k}$, with $k=2,1,-1$. Since we assume $z \neq 0$ the only possibility for $b$ to be an integer is $k=-1$ and $z^{2}=1$. This corresponds to the cases 16 and 17. We obtain $b=1$ (case 16) and $b=-1$ (case 17). Since $b=1$ yields $D=1$ we ignore this case. The other case yields $B=-3, D=3, z=e_{1}$, $y=(1-b) e_{2}=2 e_{2}$ and $w=e_{2}$, where $e_{1}, e_{2} \in\{ \pm 1\}$. Using Table 5 we find the system $\left(x-3 / 2 e_{1}\right)^{2}=1 / 4,\left(x-2 e_{1}\right)^{2}=1$, which yields $x=e_{1}$. Again the corresponding $\epsilon$ is a unit.

TABLE 8. Solutions for $\epsilon$. (mixed signs)

\begin{tabular}{|c|c|c|}
\hline$B$ & $\bar{D}$ & $\epsilon$ \\
\hline $2 n^{2}+1$ & $n^{4}+n^{2}+1$ & $\begin{array}{c} \pm n \pm \alpha \\
\pm\left(n^{3}+n-n \alpha^{2}\right) \pm\left(\left(n^{2}+1\right) \alpha-\alpha^{3}\right) \\
\pm\left(|n|^{3}-|n| \alpha^{2}\right) \pm\left(n^{2} \alpha-\alpha^{3}\right)\end{array}$ \\
\hline $2 n^{2}-1$ & $n^{4}-n^{2}+1$ & $\begin{array}{c} \pm n \pm \alpha \\
\pm\left(n^{3}-n-n \alpha^{2}\right) \pm\left(\left(n^{2}-1\right) \alpha-\alpha^{3}\right) \\
\pm\left(n^{3}-n \alpha^{2}\right) \pm\left(n^{2} \alpha-\alpha^{3}\right)\end{array}$ \\
\hline$-6 n^{2}+1$ & $9 n^{4}-3 n^{2}+1$ & $\begin{array}{c} \pm\left(6 n^{3}-n+n \alpha^{2}\right) \pm \alpha \\
\pm\left(3 n^{3}+n+n \alpha^{2}\right) \pm\left(\left(3 n^{2}-1\right) \alpha+\alpha^{3}\right) \\
\pm\left(3 n^{3}-2 n+n \alpha^{2}\right) \pm\left(3 n^{2} \alpha+\alpha^{3}\right)\end{array}$ \\
\hline$-6 n^{2}-1$ & $9 n^{4}+3 n^{2}+1$ & $\begin{array}{c} \pm\left(6 n^{3}+n+n \alpha^{2}\right) \pm \alpha \\
\pm\left(3 n^{3}-n+n \alpha^{2}\right) \pm\left(\left(3 n^{2}+1\right) \alpha+\alpha^{3}\right) \\
\pm\left(3 n^{3}+2 n+n \alpha^{2}\right) \pm\left(3 n^{2} \alpha+\alpha^{3}\right)\end{array}$ \\
\hline-3 & 3 & $\begin{array}{c} \pm 1 \pm\left(\alpha+\alpha^{3}\right) \\
\pm\left(2+\alpha^{2}\right) \pm \alpha \\
\pm\left(1+\alpha^{2}\right) \pm\left(2 \alpha+\alpha^{3}\right)\end{array}$ \\
\hline
\end{tabular}




\section{Two Corollaries}

In this section we want to prove two corollaries. The first corollary treats the case of purely quartic complex fields and the second corollary treats rings of the form $\mathbb{Z}[\alpha]$ with $\alpha=\sqrt{a}+\sqrt{b}$.

Corollary 6.1. Let $0<D \in \mathbb{Z}$ and let $\alpha$ be a root of $X^{4}+D$. The ring $\mathbb{Z}[\alpha]$ is generated by its units if and only if $D=1$.

Proof. Due to Theorem 1.2 , if $\mathbb{Z}[\alpha]$ is generated by its units, then $D=$ \pm 1 or $B^{2}-4 D=-4 D=-4,-3$. In any case we deduce either $D=1$ or a contradiction.

Corollary 6.2. Let $a, b \in \mathbb{Z}$ such that $a<0$ or $b<0$, both not squares and assume $\mathbb{Q}(\sqrt{a}+\sqrt{b})$ is quartic. Then the ring $\mathbb{Z}[\sqrt{a}+\sqrt{b}]$ is not generated by its units except if $a=b \pm 1$.

Proof. First, we note that the field $\mathbb{Q}(\alpha)$ with $\alpha=\sqrt{a}+\sqrt{b}$ is totally complex. Next we compute the minimal polynomial of $\alpha$. Since the conjugates of $\alpha$ are $\sqrt{a}+\sqrt{b}, \sqrt{a}-\sqrt{b},-\sqrt{a}+\sqrt{b},-\sqrt{a}-\sqrt{b}$, we find that $X^{4}-2(a+$ b) $X^{2}+(a-b)^{2}$ is the minimal polynomial of $\alpha$ and with $B=2(a+b)$ and $D=(a-b)^{2}$ we find $B^{2}-4 D=4(a+b)^{2}-4(a-b)^{2}$. If $\mathbb{Z}[\alpha]$ is generated by its units either $D= \pm 1$ or $B^{2}-4 D=-4,-3$. The first case yields $a=b \pm 1$ Since $4 \mid B^{2}-4 D$ it remains to investigate the equation $(a+b)^{2}-(a-b)^{2}=-1$ or equivalently $4 a b=-1$. Since $a, b \in \mathbb{Z}$ the last equation yields a contradiction.

\section{REFERENCES}

[1] N. Ashrafi and P. Vámos, On the unit sum number of some rings, Q. J. Math. 56 (2005), 1-12.

[2] P. Belcher, Integers expressible as sums of distinct units, Bull. Lond. Math. Soc. 6 (1974), 66-68.

[3] P. Belcher, A test for integers being sums of distinct units applied to cubic fields, J. Lond. Math. Soc. (2) 12 (1976), 141-148.

[4] A. Filipin, R. Tichy, and V. Ziegler, The additive unit structure of purely quartic complex fields, to appear in Funct. Approx. Comment. Math.

[5] B. Goldsmith, S. Pabst, and A. Scott, Unit sum numbers of rings and modules, Q. J. Math. Oxford Ser. (2) 49 (1998), 331-344.

[6] B. Jacobson, Sums of distinct divisors and sums of distinct units, Proc. Am. Math. Soc. 15 (1964), 179-183.

[7] M. Jarden and W. Narkiewicz, On sums of units, Monatsh. Math. 150 (2007), 327332.

[8] J. Śliwa, Sums of distinct units, Bull. Acad. Polon. Sci. Sér. Sci. Math. Astronom. Phys. 22 (1974), 11-13.

[9] R. Tichy and V. Ziegler, Units generating the ring of integers of complex cubic fields, Colloq. Math. 109 (2007), 71-83.

[10] P. Vámos, 2-good rings, Q. J. Math. 56 (2005), 417-430.

[11] L. C. Washington, Introduction to cyclotomic fields, Graduate Texts in Mathematics 83, Springer-Verlag, New York, 1997. 
[12] D. Zelinsky, Every linear transformation is a sum of nonsingular ones, Proc. Am. Math. Soc. 5 (1954), 627-630.

V. Ziegler

Graz University of Technology

Institute for analysis and computational number theory,

Steyrergasse 30,

A-8010 Graz

Austria

E-mail: ziegler@finanz.math.tugraz.at

Received: 18.11.2007.

Revised: 3.3.2008. 